Esta sección está destinada a difundir las actividades académicas de la Sociedad Chilena de Enfermedades Respiratorias, de otras Sociedades afines y de distintos centros de la especialidad, así como noticias de interés médico, científico o cultural en general. También acoge las cartas al editor, comentarios y sugerencias de los lectores y toda colaboración que se encuadre dentro de estos objetivos. El Comité Editorial se reserva el derecho de extractar, resumir y titular las cartas que se publiquen, sustrayéndose a cualquier debate con sus corresponsales.

\title{
SOCIEDAD CHILENA DE ENFERMEDADES RESPIRATORIAS
}

\section{CONGRESO CHILENO DE ENFER- MEDADES RESPIRATORIAS}

Entre el 3 y 6 de Noviembre, 2004 se celebró en Viña del Mar el congreso anual de la especialidad con 360 profesionales inscritos: La coordinación general de este congreso estuvo a cargo de las Dras. María Teresa Parada C. (medicina de adultos) y María Genoveva Parra O. (pediatría). Actuaron como coordinadoras locales las Dras. Juana Pavié G. (medicina de adultos) y Aída Milinarsky T. (pediatría). En este congreso se presentaron 25 trabajos en sesiones de comunicaciones libres y 55 como posters. Los cursos pre-congreso tuvieron una asistencia de 150 personas: el de adultos, coordinado por el Dr. Felipe Aller R. se enfocó a enfermedades obstructivas e infecciones respiratorias y el pediátrico, coordinado por la Dra. María Angélica Palomino, se dedicó a asma e infecciones pulmonares. Como conferencistas extranjeros invitados participaron ocho destacados especialistas los Drs. Richard Beaslay (Nueva Zelandia), Sonia Buist, Paul Gleezen y Douglas Wood de EE.UU., Sailesh Kotecha y Paul A. Corris de Inglaterra, Nestor Muller de Canadá y Maximiliano Van Kooten de Argentina. Se efectuó, además, un curso para pacientes de EPOC que reunió a 55 asistentes con una participación llamativamente activa.

\section{PREMIO AL MEJOR TRABAJO PRESENTADO AL CONGRESO}

De acuerdo al dictamen de la Comisión Científica recibió el Premio Sociedad Chilena de En- fermedades Respiratorias, patrocinado por el Laboratorio Glaxo-SmithKline, el siguiente trabajo:

- Etiología de la neumonia adquirida en la comunidad en adultos hospitalizados en Chile: implicancias para las guias clinicas, cuyos autores son: Alejandro Díaz, Gino Fuentes; Bernardita Couble, Reinaldo Uribe, Gesma Mercado, Alejandra Soza, Paulina Barria, Jorge Dreyse y Fernando Saldías. Este estudio está publicado en la Sección Trabajos Originales de este número de la revista (pág. 24).

\section{CONCURSO PROYECTOS DE INVESTIGACIÓN}

Por tercer año consecutivo la Sociedad otorgó un premio de apoyo financiero a los mejores proyectos presentados a este concurso. Resultaron seleccionado los siguientes proyectos:

- Utilidad diagnóstica de la detección del antígeno urinario de $S$. pneumoniae en la neumonía adquirida en la comunidad. Investigador Principal: Dra. Paulina Barría Pérez. Co-investigador: Dr. Alejandro Díaz Fuenzalida.

- Evaluación de los pacientes con hipertensión pulmonar primaria y su respuesta a sildenafil. Investigadora principal: Dra. Mónica Zagolín B. Co-investigadores: Drs. Eduardo Wainstein, Claudio Parra y Polenzi Uriarte.

CALENDARIO DE ACTIVIDADES 2005

\section{Jornadas de Patología del Sueño}

Santiago, 6 y 7 de Mayo

Invitado: Dr. Joaquín Durán Cantolla - España. 


\section{Jornadas de Invierno}

Santiago, 10 y 11 de Junio

"Alergia y compromiso respiratorio"

Invitados confirmados: Drs. Allan Becker y Anthony Frew.

\section{Congreso Chileno de Enfermedades Respiratorias}

Termas de Puyehue-Osorno. 2 al 5 de Noviembre 2005.

Invitados confirmados: Drs. Peter Barnes y Paul Jones.

\section{INFORMACIÓN COMITÉ CIENTÍFICO}

Fecha de presentación de trabajos al congreso: $1^{\circ}$ de Agosto 2005.

Fecha de concurso pre-proyectos SER: 3 de Junio 2005.

\section{NUEVOS SOCIOS}

En el último trimestre se han incorporado los siguientes nuevos socios a quienes damos una cálida bienvenida:

Dr. Alvaro Fernández G.

Dr. Andrés Koppmann A.

Dr. Franklin Mendieta F.

E.U. Edison Quiñonez C.

Dr. Luis Enrique Vega B.

\section{NUEVO SISTEMA DE PAGO DE CUOTAS SOCIETARIAS}

Se han incorporado 29 socios al servicio pago automático de su cuenta corriente (PAC) que ha significado una importante comodidad administrativa.

\section{PAGINA WEB}

La página de la Sociedad se ha ido perfeccionando gradualmente. A quienes aun no la conocen se les recomienda acceder a ella en www. serchile.cl, donde encontrará toda la información de futuras actividades con sus respectivos programas y fichas de inscripción. Además puede acceder a:

- Conferencias de las jornadas de invierno 2004 y congreso 2004 en "Ver presentaciones"

- Links a revistas internacionales

- Link a Revista Chilena de Enfermedad Respiratorias
- Búsqueda de trabajos presentados a los congresos

- Información de congresos internacionales

- Indice de la Revista Chilena de Enfermedades Respiratorias desde 1985

- Consejos y trucos para manejar su PC

Para acceder a algunas secciones se le pedirá su nombre y contraseña. El nombre se estructura en minúsculas anotando primero la inicial del nombre, seguida del apellido y la inicial del segundo apellido. La contraseña son los primeros cuatro números del 212 7684-7. En caso de problemas dirigirse a la secretaría de la Sociedad (231 6292).

$\mathrm{Si}$ algún socio/a estima que existe o él/ella tiene alguna información que debiera estar en la página web rogamos hacer llegar su proposición a la Sociedad para que lo revise el Comité Editorial de la página, a cargo del Dr. Raúl Corrales $\mathrm{V}$.

\section{AÑOS DE VENTILACIÓN MECÁNICA NO INVASIVA EN EL INSTITUTO NACIONAL DEL TÓRAX}

\section{Curso Taller de Ventilación Mecánica No Invasiva (VNI)}

Fecha: 4 y 5 de agosto, 2005.

Lugar: Hotel Intercontinental (Santiago)

Invitado extranjero:

Nicholas Hill (Rhode Island Hospital)

Invitados nacionales

Dr. Felipe Aller (Instituto Nacional del Tórax)

Dr. Guillermo Bugedo (Universidad Católica)

Sr. Pablo Zapararte (Ingeniero Biomédico)

Dr. Luis Soto (Instituto Nacional del Tórax)

Dr. Sergio Valdés (Clínica Las Condes)

Temas:

VNI en patología respiratoria aguda y crónica.

VNI en patología cardiovascular aguda y crónica.

VNI en falla respiratoria aguda.

Director:

Dr. Cesar Maquilón

Coordinadores:

Drs. Matías Florenzano y Cristian Ibarra

Inscripciones Secretaría Docente:

Sra. Judith Molina

Fono: 3403505

José Miguel Infante 717. Primer piso. 


\section{CARTA AL EDITOR}

\section{Protocolo: Evaluación de la respuesta clínica, funcional y hemodinámica a la terapia con Sildenafil en los pacientes con hipertensión pulmonar arterial}

Señor editor:

En el Instituto Nacional del Tórax se está llevando a cabo la evaluación de pacientes con Hipertensión Pulmonar Arterial no vasorreactivos (test de Adenosina negativo), se propone la terapia con Sildenafil.

Los criterios de inclusión en este protocolo son los siguientes:

- Pacientes con Hipertensión Pulmonar Arterial primaria o idiopática o asociada a mesenquimopatías, VIH, drogas.

- Pacientes sin evidencias de falla ventricular izquierda o daño pulmonar parenquimatoso.
- Pacientes mayores de 18 años que se encuentren sin terapia específica para su hipertensión pulmonar.

- Pacientes con posibilidades de concurrir a controles mensuales y en forma precoz y oportuna en caso de deterioro de su condición.

Los pacientes que reúnan estas condiciones pueden ser referidos al Policlínico de Hipertensión Pulmonar de los días viernes entre las 10:00 y 12:00 h en el Instituto Nacional del Tórax para una primera evaluación.

Se agradece la gentil colaboración en esta grave y compleja condición que afecta principalmente a pacientes jóvenes impactando seriamente en su calidad de vida y sobrevida.

Dra. Mónica Zagolín B. E-mail: mzagolin@torax.cl Fono: 3403505

\section{NOMBRAMIENTO DE MIEMBROS HONORARIOS}

En una sesión plenaria especial del Congreso se dió a conocer el acuerdo unánime del Directorio de distinguir con nombramiento de miembros honorarios los Drs. Carmen Lisboa B., Patricia Díaz A., Edgardo Carrasco C. y Manuel Oyarzún G., en reconocimiento a su destacada trayectoria dentro de la Sociedad.

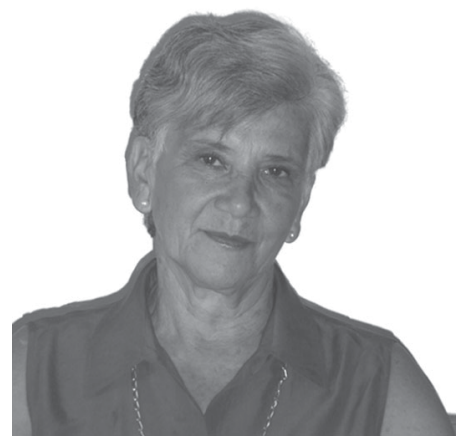

La Dra, Gisella Borzone se refirió a los méritos de la Dra. Carmen Lisboa Basualto que justificaron esta distinción. La Dra. Lisboa estudió Medicina en la Universidad de Chile, obtuvo su título en 1964 y fue la primera becaria de la especialidad en la Universidad Católica, a la cual ingresó luego como Instructor Asociado en 1966. En 1977 obtuvo el nombramiento de Profesor Adjunto y el de Profesor Titular de Medicina en 1988. Su formación en Canadá, en la Universidad Mc Gill de Montreal, orientó su vocación de investigadora. Además de ser Jefe del Departamento de Enfermedades Respiratorias por 11 años, llegó a ser Directora de Investigación de la Facultad y el año 2003, la Facultad de Medicina en reconocimiento a su destacada labor, le otorgó el premio a la Trayectoria en Investigación. Uno de sus logros como Directora de investigación fue el programa de inmersión de verano en laboratorios de investigación para alumnos de los primeros años de Medicina, gracias al cual, varios de ellos han presentado trabajos originales en ésta como en otras sociedades científicas.

Su actividad científica se inició con el trabajo de ingreso a la entonces Sociedad Chilena de Enfermedades del Tórax y Tuberculosis, con el trabajo titulado: "Mecánica ventilatoria y control de la respiración en el pulmón senil”, realizado con el estímulo y colaboración del Dr Ricardo Ferretti. Hoy ha acumulado más de un centenar de publicaciones en revistas de corriente principal tanto nacionales como extranjeras. El desarrollo de su línea de investigación le ha significado el reconocimiento de sus pares a nivel internacional y es así como se le ha solicitado 
compartir su experiencia en capítulos de libros señeros de la especialidad. Participó en la fundación de la Asociación Latinoamericana del Tó$\operatorname{rax}$ (ALAT) en 1996, llegando a ser su presidente en el período 1998 a 2000.

Desde el inicio de su vida profesional, la Dra Lisboa se incorporó a nuestra Sociedad siendo numerosas sus contribuciones: formó parte del Directorio en dos oportunidades, ha contribuido de manera significativa con trabajos originales en nuestros congresos y Revista y ha compartido su experiencia tanto en la Comisión Científica como en su calidad de editor asociado de la Revista Chilena de Enfermedades Respiratorias.

Para la Dra. Lisboa, como para muchos de nosotros, las Sociedades existen para lograr entre todos lo que para cada uno individualmente es difícil conseguir y es así como se entregó con gran entusiasmo y dando lo mejor de sí para elevar el nivel científico de nuestra Sociedad, caracterizándose por la vehemencia con que plantea sus críticas y hace sugerencias demostrando así el interés que tiene por el tema como de su preocupación por el desarrollo de las personas.

Es así, como la doctora ha tenido una especial vocación por la formación de numerosos becarios, que hoy ejercen a lo largo de todo Chile, quienes recordarán como la presión y ayuda de la Dra. Lisboa fue esencial para salir adelante con las publicaciones que generaron. Vale la pena destacar que y aún cuando detrás de la idea, impulso y gestación del manuscrito estaba la mano de la Dra. Lisboa, en todas ellas figuraba el becario como primer autor, una muestra de generosidad que merece el mayor reconocimiento.

Desde hace algún tiempo, 7 nietos compiten con sus intereses académicos, para quienes el mejor de los panoramas es ir a la parcela de la “abuela Carmen", donde se la puede ver en cuclillas desmalezando sus macizos de flores o cosechando paltas, con el mismo entusiasmo con que desarrolla sus labores académicas. Quizás la prueba más fehaciente de esta dedicación es que en contra de lo que nos enseñara la Dra. Paulina Ridemann en Puyehue, la Dra. Lisboa logró hacer crecer y florecer un notro austral en su parcela de Santo Domingo.

Es de esperar que la Dra. Lisboa continúe sembrando semillas de cariño por la investigación en la Universidad y en esta Sociedad y que las lleve a florecer, aún más frondosamente que su notro, en los años venideros.

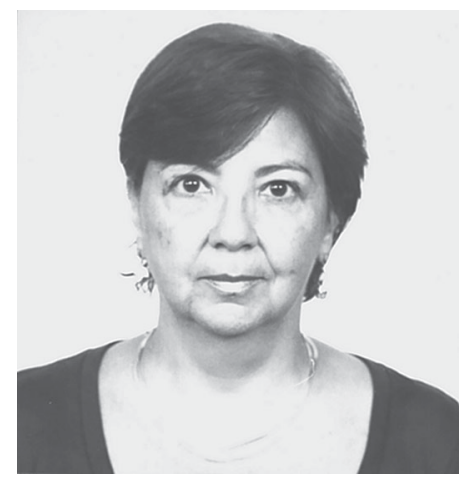

La semblanza de la Dra. Patricia Díaz estuvo a cargo del Dr. Ricardo Pinto quien se expresó en los siguientes términos: "La Dra Díaz ha sido elegida Socia Honoraria en mérito no sólo a su extensa y exitosa carrera sino por la entrega generosa de sus conocimientos, de su tiempo y de su valiosa experiencia como ser humano.

La Dra. Díaz inicia su carrera profesional en la Escuela de Medicina de la Universidad de Chile entre los años 1960 y 1968 e inmediatamente inicia su formación como pediatra en el
Hospital Manuel Arriarán. En 1970, la Universidad de Chile ve en ella un médico capaz de constituirse en un futuro académico, y es así que le otorga una beca de formación clínico básico, completándola a fines de 1973. Los trágicos tiempos vividos por Chile no la alejaron de sus valores fundamentales, por lo que continuó estudiando y trabajando como siempre y preparándose para uno de sus más trascendentales pasos: realizar estudios en inmunología respiratoria en la prestigiosa Universidad de Edimburgo, entre 1975 a 1979. Este gran esfuerzo lo coronó con la obtención de un doctorado $(\mathrm{PhD})$, convirtiéndose en uno de los primeros médicos chilenos en obtener este prestigioso grado académico. Pasó casi un año antes de que se decidiera volver a Chile, incorporándose al Departamento de Medicina Experimental, de la Facultad de Medicina de la Universidad de Chile, en el que trabaja hasta estos días. El conocimiento adquirido lo volcó rápidamente en nuestro medio, presentándonos su trabajo previo y exponiéndonos al desconocido macrófago alveolar, como una de las células más importantes de la patología pulmonar. La Dra. Díaz fue además una de las primeras investigadoras que de manera 
directa nos introdujo en los mecanismos íntimos de la inflamación y de los hasta ese momento indescriptibles e incomprensibles mecanismos inmunológicos. Nunca ha abandonado la clínica, constituyéndose en uno de los puentes más sólidos entre el conocimiento del mesón de laboratorio y la cama del enfermo. Cuando la relación asma e inmunología comenzaron a no responder las interrogantes del mundo científico, ella fue nuevamente pionera en el desarrollo del conocimeinto entre infecciones virales y bronquitis obstructiva, como un paso inicial en el desarrollo del asma. Actualmente este proyecto la ha hecho conocida internacionalmente y ocupa la mayor parte de su tiempo.

Patricia también ha tenido tiempo para las actividades científicas de varias sociedades, sin embargo, es en la Sociedad Chilena de Enfer- medades Respiratorias donde ha ocupado casi todos los puestos de responsabilidad, hasta llegar a haber sido una de sus más brillantes presidentes.

Hoy día tiene a su cargo el Comité Científico, convirtiéndolo en motor y verdadera alma de nuestra Sociedad.

Finalmente, no quisiera dejar fuera el rol esencial de la mujer, el ser madre. En él también ha sido un ejemplo, donde cada uno de nosotros ha visto reflejada el coraje, la valentia y su incansable fuerza para salir adelante.

La Sociedad toda, se enorgullece en entregarle este pequeño homenaje, pero en el corazón de cada uno de nosotros le entregamos nuestro agradecimiento, por haber sido un ejemplo de lo que significa ser mujer, madre y abnegada pediatra".

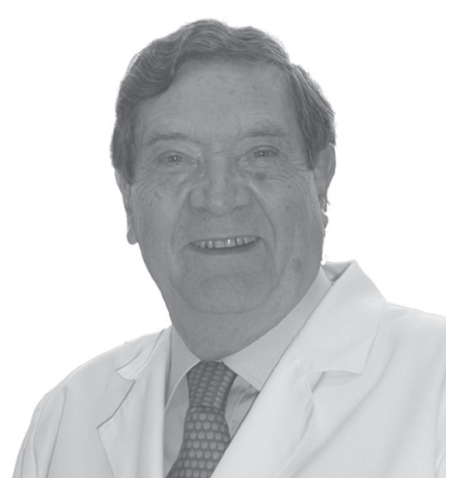

El Dr. Manuel Oyarzún se refirió a la carrera y personalidad del Dr. Edgardo Carrasco Calderón recordándolo como su profesor de Tisiología caracterizado por su gran capacidad de trabajo, su perseverancia y su calidad académica. El Dr. Carrasco nació en Iquique, cursó sus estudios de enseñanza básica en Iquique y en $\mathrm{La}$ Serena y completó su enseñanza media en el Instituto Nacional de Santiago

En 1946, ingresó a la Escuela de Medicina, Universidad de Chile, titulándose de médico-cirujano en 1953 e ingresó al Servicio de Medicina del Profesor Rodolfo Armas Cruz. Ese mismo año el Dr. Carrasco da un paso trascendental en su vida al contraer matrimonio con la Srta. Angela Casals Gimeno.

En 1956, nace el Depto. de Enfermedades Broncopulmonares del hospital San Juan de Dios cuyos únicos médicos son los Drs. Victorino Farga y E. Carrasco.

Dos años después el Dr. Carrasco viajó a la la Universidad de Harvard en Boston con una Beca de la Fundación Doherty, donde estudió Clínica de Enfermedades Respiratorias y función pulmonar con el Dr. John Knowles, y Alergia e Inmunología de Enfermedades Respiratorias con el Dr. Francis Lowell.

A su regreso a Chile, organizó con el Dr. Victorino Farga el Centro de Enfermedades Broncopulmonares del hospital San Juan de Dios, donde trabajaron en sus inicios los Drs Gladio Mena, Alvaro Yáñez, Isidoro Busel y Osvaldo Koller. Allí se consolidó la atención de la TBC a nivel ambulatorio con la formación de la primera central de tratamiento ambulatorio de la tuberculosis con que contó el país.

En 1967, el Dr. Carrasco sucedió al Dr. V. Farga en la dirección del Centro de Enfermedades Broncopulmonares y, además, asumió como sub-jefe del Servicio de Medicina del hospital San Juan de Dios desempeñando ambos cargos hasta 1974. Sus méritos académicos lo acreditaron en 1971 como profesor titular de Medicina de la Universidad de Chile, realizando una intensa actividad docente tanto a nivel de pregrado como de postgrado dirigiendo becas de formación en Neumotisiología

En 1974, es nombrado director del Instituto Nacional de Enfermedades Respiratorias y Cirugía Torácica, cargo que ejerció, durante 15 años. Bajo su dirección el INERyCT experimentó una reorganización de sus actividades institucionales con una intensa actividad docente de pre y post grado, llegando el Instituto a contar con 20 becados simultáneos en Enfermedades Respiratorias y Cirugía Torácica. El Dr. Carrasco estimuló la 
realización de múltiples cursos de postítulo y Ciclos de Conferencias como Inmunología y Pulmón, Avances en Neumología, Cuidados Intensivos y Cirugía Torácica, muchos de los cuales aun continúan realizándose.

En esta misma época el Dr. Carrasco lideró en calidad de editor y director la creación de la revista Enfermedades Respiratorias y Cirugía Torácica, cuyo primer número salió a circulación en 1985. Cinco años más tarde ésta se constituye en el órgano oficial de la Sociedad Chilena de Enfermedades Respiratorias.

Luego de haber dejado la dirección del INERYCT en 1989, el Dr. Carrasco continuó a cargo de la docencia del Instituto hasta el año 2000 en que se acogió a retiro, aunque ha mantenido su actividad como profesor titular de Medicina de la Universidad de Chile.

Presidió la Sociedad Chilena de Enfermedades Respiratorias (1990-1991), la Sociedad Chi- lena de Alergia e Inmunología en dos períodos (1983-4 y 2002-3), la Comisión Nacional de Calificación de Especialistas en Enfermedades Respiratorias (CONACEM) desde 1984 hasta ahora y el Comité de evaluación de Centros formadores de especialistas en enfermedades respiratorias de la Asociación de Facultades de Medicina de Chile (ASOFAMECH) desde 1985 hasta ahora.

Más de 100 publicaciones en revistas nacionales e internacionales dan testimonio de su experiencia académica y profesional. Destacando en los últimos años su participación en la creación de GINA (Iniciativa Global para el Asma) y en la redacción de los documentos internacionales emanados de esta institución. Recientemente encabezó la Comisión de Asma que redactó las Guías para el Diagnóstico y Manejo del Asma de esta Sociedad y que han sido publicadas en el tercer número de 2004 de la Revista Chilena de Enfermedades Respiratorias.

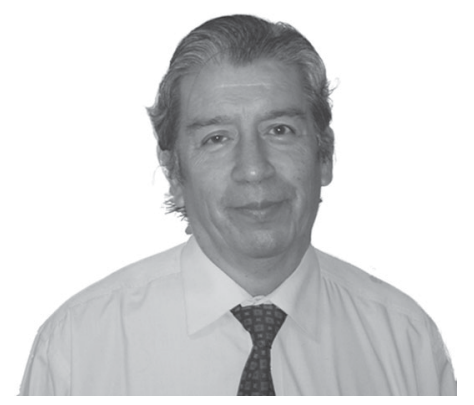

La descripción de la trayectoria y perfil humano del Dr. Manuel Oyarzún Gómez fue realizada por el Dr. Sergio Bello.

El Dr. Oyarzún nació en Santiago, hijo del Dr. Manuel Oyarzún Palominos, un destacado cirujano de la Asistencia Pública y del hospital San Juan de Dios. Está casado desde hace más de 30 años con Paulina Sanhueza Vargas, con descendencia de dos hijos. Estudió las humanidades en el liceo de hombres $\mathrm{N}^{\circ}$ 4, Manuel Barros Borgoño. En 1960 ingresa a estudiar medicina en la Universidad de Chile. Recién egresado, ingresa al hospital del Tórax, donde recibe una sólida formación clínica bajo la tuición del Prof. Victorino Farga y paralelamente se forma en fisiología y fisiopatología respiratoria en el departamento de Medicina Experimental de la Universidad de Chile, con el Prof Elías Motles. Muy tempranamente se destaca por su espíritu emprendedor y a poco andar colabora con el Dr. Rodolfo Paredes en la formación de la Unidad de Cuidados Intensivos del hospital del Tórax.

En aquella época nace su pasión por el surfactante pulmonar, la que se consolida y profundiza durante su programa de post-doctorado que realiza en el Cardiovascular Research Institute de la Universidad de San Francisco California, trabajando con el Dr. John Clements, máxima autoridad mundial en la materia con el que permaneció durante cuatro años, 19741978.

Por sus aportes en esta línea de trabajo y en función pulmonar ha sido invitado a dictar numerosas conferencias y como profesor visitante a la Universidad de British Columbia, en Canadá y a diversos países latinoamericanos como Argentina, Uruguay, Bolivia y México. Su afán de investigador lo han llevado a lugares muy especiales como lo fue una investigación sobre función pulmonar en la Antártica. Ha publicado más de 140 artículos y capítulos de libros, en diversas revistas especializadas y de prestigio en nuestro país y en el extranjero.

Es profesor titular de la Universidad de Chile desde 1987, desempeñando una incansable actividad de pre y post grado, organizando un sinnúmero de cursos entre los que destaca haber sido director de doce cursos anuales de Fisiopatología Respiratoria realizados ininterrumpidamente entre los años 1979 y 1990 en el Instituto Nacional del Tórax, con el auspicio de la 
Escuela de Post-grado de la Facultad de Medicina de la Universidad de Chile.

Desde fines de los ' 80 y comienzos de los ' 90 se apasionó por otro tema: el medio ambiente, la contaminación y sus efectos sobre la salud y el aparato respiratorio, realizando numerosas investigaciones al respecto. También participó en la creación del Magister en medio Ambiente y Biomedicina y del Centro de Investigaciones del Medio Ambiente de la Facultad de Medicina de la Universidad de Chile, del cual es su director desde 1995 a la fecha, además de ser el representante de la Facultad ante el Comité Ejecutivo del Centro Nacional del Medio Ambiente.

En la Facultad de Medicina ha desempeñado numerosos cargos, siendo en la actualidad Director del Programa de Fisiopatología del Instituto de Ciencias Biomédicas, destacando sin duda haber sido Director Académico de la Facultad de Medicina entre los años 1994 y 1998 desde donde promovió la creación del Programa de Doctorado en Ciencias Médicas.

En nuestra Sociedad Chilena de Enfermedades Respiratorias, es socio desde 1968 en adelante y fue Presidente en el período 1982-1983 y Secretario General entre 1990 y 1991. Además es coordinador de la Comisión de Contaminación Ambiental desde 1992, miembro del Comité Científico y representante de la sociedad ante CONACEM desde su creación en 1984. Pero sin duda su mayor contribución a nuestra sociedad ha sido su tesonero e incansable trabajo al frente de la Revista Chilena de Enfermedades Respiratorias participando desde la creación de su precursora, la revista Enfermedades Respiratorias y Cirugía Torácica, y es Editor-Director de la actual revista desde 1989 hasta la fecha. Existe consenso que si no fuera por Manuel la revista no existiría. 